\title{
EchoGéo
}

11 | 2010

Madagascar

\section{Interview de Jean-François Drevet}

Alexis Sierra

\section{OpenEdition}

Journals

Édition électronique

URL : https://journals.openedition.org/echogeo/11634

DOI : 10.4000/echogeo. 11634

ISSN : 1963-1197

\section{Éditeur}

Pôle de recherche pour l'organisation et la diffusion de l'information géographique (CNRS UMR 8586)

Référence électronique

Alexis Sierra, «Interview de Jean-François Drevet », EchoGéo [En ligne], 11 | 2010, mis en ligne le 24 février 2010, consulté le 31 juillet 2021. URL : http://journals.openedition.org/echogeo/11634 ; DOI : https://doi.org/10.4000/echogeo.11634

Ce document a été généré automatiquement le 31 juillet 2021.

EchoGéo est mis à disposition selon les termes de la licence Creative Commons Attribution - Pas d'Utilisation Commerciale - Pas de Modification 4.0 International (CC BY-NC-ND) 


\title{
Interview de Jean-François Drevet
}

\author{
Alexis Sierra
}

1 Géographe, Jean-Francois Drevet a été responsable de la prospective, puis de l'élargissement, à la Direction Générale, de la politique régionale de la Commission Européenne à Bruxelles de 1989 à 2005.

2 - Alexis Sierra (AS). Monsieur Jean-François Drevet, bonjour. Agrégé de géographie issu de l'Ecole Normale Supérieure, vous avez eu un parcours professionnel atypique: Université, filiale de la Caisse des Dépôts et Consignation, Ministère de l'Agriculture, DATAR, cabinet ministériel et depuis 1989, Commission Européenne au sein de la Direction Générale de la politique régionale (DG Regio). Quel a été le rôle de vos compétences de géographe dans ce parcours?

3 - Jean-François Drevet (JFD). Ma formation de géographe a été très utile dans ces différents postes. Quand j'étais étudiant, la géographie régionale était une sousdiscipline majeure qui développait la capacité de synthèse entre données issues de différentes disciplines et sur un territoire donné. Les cours de Jacqueline BeaujeuGarnier à l'Institut de Géographie sur l'Aménagement du territoire ont été un révélateur et m'ont servi de fil rouge. L'esprit de cette formation a été déterminant dans les activités que j'ai menées par la suite que ce soit pour planifier le développement de la Côte d'Ivoire dans les années soixante-dix ou préparer l'adhésion de l'Espagne et du Portugal, puis de la Pologne et des autres pays d'Europe centrale à l'Union Européenne ou encore faire des propositions sur la Turquie au sein de la Commission européenne.

4 En revanche, je n'ai pas été recruté en qualité de géographe; cela n'a pas été le déclencheur des nominations. Ce parcours tient à mon désir initial de travailler comme expert dans le cadre de la coopération avant de faire éventuellement une carrière d'enseignant. A partir de mon service national en coopération qui m'a conduit en Afrique, c'est cette logique d'expertise qui s'est enclenchée. J'ai été recruté au ministère de l'agriculture dans la perspective de l'adhésion de l'Espagne à la CEE parce que le dossier était impopulaire auprès de la profession ; j'ai été recruté à la DATAR à la suite de cette expérience dans le développement rural et agricole européen. A la DATAR, je m'occupais des fonds structurels communautaires ce qui m'a permis d'entrer à la DG 
Regio de la Commission Européenne pour lancer un programme de prospective territoriale, puis de mettre cette prospective en application avec la préparation des pays candidats à leur adhésion.

5 Au final, l'Aménagement du territoire a été un fil directeur.

6 - AS. La Commission européenne recrute-t-elle directement des géographes?

7 - JFD. A la DG Regio, je n'ai croisé que peu de géographes, quatre ou cinq au total, anglais, allemand, hollandais et espagnol. On en trouve aussi à la DG environnement et certainement ailleurs. La majorité des fonctionnaires européens est recrutée sur concours général de type ENA. Les concours spécialisés concernent des activités particulières comme les statistiques ou l'audit. Or, il n'existe pas d'épreuve de géographie stricto sensu ou d'option pour géographe. Les études de géographie offrent une culture générale, une pratique de la synthèse, utile mais il n'y a pas de recrutement spécifique de géographes. En revanche, l'analyse géographique est une compétence demandée pour la politique régionale.

8 - AS. La DG Regio fait-elle alors appel à des géographes en dehors de la Commission?

9 - JFD. Oui. La Commission fait appel à des consultants qui relèvent soit de bureaux d'étude généralistes soit de laboratoires universitaires. Les bureaux d'études privés ont davantage l'habitude de répondre à des appels d'offres dont le format est de plus en plus contraignant; ils ont une communication plus offensive, intègrent plus rapidement les normes requises. En revanche, les universitaires sont moins coûteux, plus indépendants et plus disponibles pour approfondir les analyses. En effet, souvent les études issues de bureaux d'étude n'osent pas être critiques pour ne pas prendre de risque vis à vis de clients potentiels. Avec les universitaires, j'ai pu établir un dialogue pour approfondir telle ou telle analyse au-delà du cadre établi par l'appel d'offre. La vision prospective est plus stimulante. Nous encadrons également des stagiaires.

10 - AS. Quelles sont les études demandées?

11 - JFD. Nous avons besoin d'expertise en particulier pour l'évaluation de chacun des programmes opérationnels et des projets visant à obtenir des financements communautaires. Nous faisons alors appel à des équipes internationales où on trouve des géographes et à des économistes, les premiers inscrivant les évaluations et la prospective dans une logique territoriale concrète. A partir des priorités de l'Union Européenne, les géographes vont hiérarchiser les projets déposés en fonction des territoires régionaux, de leur environnement national et communautaire.

12 - AS. Les constructions géographiques de type «arc » ou « diagonales » ont-elles une influence sur les priorités de la politique régionale européenne?

13 - JFD. Oui. Il y a bien une influence des géographes à ce niveau-là. Quand je suis arrivé à la Commission pour lancer un programme de prospective, il fallait trouver une légitimité transnationale aux scenarii à proposer. Il existait déjà des coopérations qui s'inscrivaient dans une représentation régionale de l'Europe comme le Nord-Ouest européen incluant le Bénélux, les îles Britanniques, le Nord de la France et une partie de l'Allemagne. Une convention alpine, incluant la Suisse et l'Autriche, était en préparation. Cependant, il fallait couvrir l'ensemble communautaire et trouver des logiques de coopération. C'est ainsi que j'ai proposé une carte avec un découpage comprenant un arc Atlantique, un arc Méditerranéen, un ensemble Baltique, un axe continental en prolongeant la « diagonale aride » de Roger Brunet. Cette carte a permis de mettre en évidence l'existence de régions marginalisées. Il y a eu des rapports 
d'étude et des consultations. Toutes les régions n'adhéraient pas à ce type de construction. Par exemple, l'Ecosse était peu intéressée par une coopération " atlantique ». Par la suite, nous avons travaillé sur un ensemble danubien et un autre autour de la Mer Noire. Ces découpages n'ont pas eu le même succès : les riverains de la mer Baltique ont mis en œuvre une coopération efficace à partir des études prospectives alors que l'arc méditerranéen n'a pas réussi à se concrétiser.

14 - AS. Comment expliquez-vous cette différence?

15 - JFD. Au niveau de la Baltique, il existait déjà une coopération entre certains pays dans le cadre du Conseil Nordique qui faisait fonctionner un bureau d'étude. Avec la fin de la Guerre froide, la réunification allemande puis les élargissements successifs de 1995 (Suède, Finlande) et de 2004 (Pologne, pays baltes), la mer Baltique est devenue un enjeu commun. Les questions environnementales ont joué en ce sens, chacun s'apercevant qu'un effort pour réduire la pollution n'était efficace que s'il était concerté.

16 - AS. Ces constructions géographiques ont-elle eu un impact concret?

17 - JFD. Elles servent à faire se rencontrer des acteurs qui parfois s'ignorent ou s'opposent. Ainsi, autour de la mer Noire, malgré les hostilités nationales, le travail sur des dossiers techniques, moins connus du grand public, peut se réaliser. Ces études prospectives ont servi de base pour créer en 1995, en complément de la coopération transfrontalière, le programme Interreg de coopération transnationale, qui oriente donc les financements du FEDER correspondant (INTERREG II-C, puis III-B et coopération territoriale européenne). Enfin, une carte des réseaux transeuropéens a permis de définir des axes prioritaires au niveau du continent tout entier.

18 Les études menées permettent aussi d'évaluer la mise en cohérence de politiques sectorielles à forte charge territoriale, comme l'environnement et les transports, et de mettre en évidence les contradictions, ne pas accepter un projet d'infrastructure qui passe par une zone classée Natura 2000 par exemple.

19 - AS. Les découpages proposés s'appuient sur le niveau régional (NUTS 2). Il en est de même des financements. La formule "l'Europe des régions" a-t-elle un sens pour vous ?

20 - JFD. Une Europe à 27 Etats-membres est difficile à faire fonctionner, alors une Europe à 250 ou 300 régions... Au niveau européen, l'échelon régional a rapidement gagné une existence. Au début des années soixante-dix, il y a eu la création d'une conférence des régions périphériques et maritimes qui a été un des premiers lobbies régionaux. Pour se rapprocher des citoyens, la Commission s'est appuyée sur le niveau régional. Le traité de Maastricht en établissant le Comité des Régions a donné un rôle aux collectivités territoriales et notamment aux régions, leur consultation étant rendue obligatoire. En revanche leur avis n'est que consultatif. Avec la décentralisation, les Etats ne peuvent pas occulter l'avis des Régions. L'influence des élus régionaux dépend des compétences acquises dans le cadre national. Les élus allemands, belges, espagnols ont de vrais pouvoirs et peuvent avoir une influence au niveau européen.

21 Cet échelon régional a pris une importance nouvelle avec les derniers élargissements, qui ont modifié l'équilibre entre les grands et les petits États, à l'avantage des seconds. Dans la mise en œuvre de leurs politiques territoriales, ces pays ont davantage besoin de concertations avec leurs voisins que les grands. Comme l'UE à 27 est désormais trop vaste, on assiste à l'émergence d'espaces transnationaux opérationnels de dimension intermédiaire, plus petits que l'UE et plus grands que les États, basés sur un 
regroupement géographique pertinent: un bassin maritime (Baltique, Mer du Nord, Atlantique) ou fluvial (le Danube), un massif montagneux (les Alpes), où peuvent être coordonnées les politiques territoriales : aménagement du territoire, environnement, transports, etc... Par exemple, la Hongrie ne peut trouver de solution à certains de ses problèmes territoriaux qu'en concertation avec ses voisins danubiens.

22 - AS. Ces études s'appuient sur une production cartographique. Où est-elle élaborée ?

23 - JFD. La DG Regio a son propre service de cartographie depuis 1995. Il travaille en priorité pour alimenter le site web et pour spatialiser les données statistiques. Les Commissaires apprécient cette production cartographique.

24 - AS. Pourquoi cet intérêt ?

25 - JFD. Les commissaires en charge de la politique régionale ne sont pas forcément des spécialistes de l'aménagement du territoire. Les cartes leur permettent de rendre plus facilement intelligible un problème.

Les cartes sont également un formidable instrument de communication. Les journalistes les apprécient et publient généralement celles que nous leur fournissons.

27 En revanche, c'est un instrument à manier avec beaucoup de prudence. La confection de cartes révèle des situations parfois en contradiction avec ce qu'avancent les acteurs nationaux ou régionaux. Nous avons souvent eu des réactions négatives. Certains de nos propos, établis à partir d'analyses cartographiques, ont pu être contestés au fait que nous étions coupés du terrain... C'est pourquoi la préparation des cartes est étroitement contrôlée, même quand elles sont prospectives.

[Entretien réalisé par Alexis Sierra] 